Research Paper

\title{
Comparison of macrovascular invasion-free survival in early-intermediate hepatocellular carcinoma after different interventions: A propensity score-based analysis
}

\author{
Yao Liu1 ${ }^{*}$, Dongying Xue ${ }^{2 *}$, Shanzhong Tan ${ }^{3^{*}}$, Qun Zhang1, Xue Yang1, Yuxin Li1, Bingbing Zhu ${ }^{4}$,

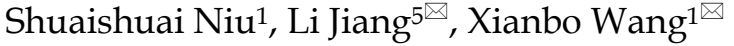 \\ 1. Center of Integrative Medicine, Beijing Ditan Hospital, Capital Medical University. Beijing 100015, China. \\ 2. Department of Infections Disease, Putuo Hospital, Shanghai University of Traditional Chinese Medicine. Shanghai 200062, China. \\ 3. Department of Integrated TCM and Western Medicine, the Second Hospital of Nanjing, Nanjing University of Chinese Medicine, Nanjing 210003, China. \\ 4. Department of Gastroenterology, Dongzhimen Hospital, Beijing University of Chinese Medicine. Beijing 100700, China. \\ 5. Department of Surgery, Beijing Ditan Hospital, Capital Medical University. Beijing 100015, China. \\ *These authors contributed equally to this work. \\ $\triangle$ Corresponding authors: Dr. Li Jiang, Department of Surgery, Beijing Ditan Hospital, Capital Medical University, No. 8 Jing Shun East Street, Beijing, 100015, \\ China. Tel: +86-010-64322407; Fax: +86-010-64322407; E-mail: 13801059895@139.com. Dr. Xianbo Wang, Center of Integrative Medicine, Beijing Ditan Hospital, \\ Capital Medical University, No. 8 Jing Shun East Street, Beijing, 100015, China. Tel: +86-010-84322301; Fax: +86-010-84322301; E-mail: wangxb@ccmu.edu.cn
}

(c) The author(s). This is an open access article distributed under the terms of the Creative Commons Attribution License (https://creativecommons.org/licenses/by/4.0/). See http://ivyspring.com/terms for full terms and conditions.

Received: 2018.09.11; Accepted: 2019.05.17; Published: 2019.07.08

\begin{abstract}
Objectives: The purpose of this study was to compare macrovascular invasion (MVI)-free survival (MFS) at the three-year follow-up in patients with hepatocellular carcinoma (HCC) who underwent hepatic resection $(\mathrm{HR})$, transcatheter arterial chemoembolization (TACE), or TACE combined with radiofrequency ablation (TACE-RFA).

Materials and Methods: We retrospectively analyzed the medical records of 828 patients who were diagnosed with Barcelona Clinic Liver Cancer (BCLC) stage A or stage B HCC. Of these patients, 116 underwent HR, 395 underwent TACE-RFA, 239 underwent TACE, and 78 patients received conservative treatment (control group). A validation cohort of 158 patients was included. The MFS and overall survival (OS) before and after propensity score (PS) matching were evaluated using Kaplan-Meier analysis.

Results: The baseline characteristics between the control and TACE groups were comparable. MFS was higher in the TACE group than in the control group at the three-year follow-up $(p=0.0091)$, and OS was similar in the two groups $(\mathrm{p}=0.0549)$. PS matching was used to generate 68 pairs of patients in the control versus $\mathrm{HR}$ group and 74 pairs of patients in the control versus TACE-RFA group ( 1 -to-1 matched). MFS was significantly higher in the HR or TACE-RFA groups than in the control group $(p<0.0001$ (HR versus control) and $p=0.0001$ (TACE-RFA versus control), respectively). Furthermore, for patients in the HR versus TACE-RFA versus TACE groups that were generated by PS matching, the Kaplan-Meier analysis showed that MFS and OS were higher with HR or TACE-RFA than with TACE at three years. In the study, similar results were obtained in the validation cohort.
\end{abstract}

Conclusions: MFS and OS were higher with HR or TACE-RFA than with TACE for HCC patients without MVI.

Key words: Macroscopic vascular invasion; Hepatocellular carcinoma; Propensity score analysis

\section{Introduction}

Hepatocellular carcinoma (HCC) is regarded as the fifth most common malignancy worldwide and the third cause of mortality related with cancer (1). In total, $10-40 \%$ of patients are diagnosed with macrovascular invasion (MVI) associated with the portal vein, hepatic vein and/or inferior vena cava 
(2-4). For patients with non-resectable HCC, MVI negatively impacts the prognosis to a large extent and leads to lower median survival (two to four months) than those with no MVI whose median survival is 10-24 months (3-5). Among the types of MVI that contribute to poor outcomes, portal vein tumor thrombus is most important $(4,6)$ as it can cause liver dysfunction, portal hypertension, ascites, variceal rupture, hepatic encephalopathy, and/or death.

According to the internationally recognized guidelines of HCC management published by the American Association for the Study of Liver Diseases (AASLD), liver transplantation is the first choice for patients with early $\operatorname{HCC}(7,8)$. Post-transplantation overall survival (OS) at 5 years among such patients can reach approximately $70 \%$ (9-11). However, in view of donor organ shortage, high cost, as well as longer waiting times for transplantation, an alternative therapeutic procedure such as hepatic resection (HR) and radiofrequency ablation (RFA) can greatly deter the progress of HCC in clinical practice (12). Moreover, two retrospective studies demonstrated that transarterial chemoembolization (TACE) combined with RFA (TACE-RFA) has an efficacy comparable to hepatectomy for primary HCC $(13,14)$. Recently, many centers have reported that TACE has emerged as an alternative to HR for treating HCC patients (15-18), with one study suggesting that OS following TACE can rival the OS reported after HR (15). The use of TACE has a wider application, as opposed to the Barcelona Clinic Liver Cancer (BCLC) staging system which recommends TACE only for patients with advanced $\operatorname{HCC}(19,20)$. Many studies have compared the therapeutic efficacy of these interventions on survival outcomes $(21,22)$. However, to our knowledge, no study has compared the effects of various treatments on MVI-free survival (MFS).

In order to compare the efficacy of $\mathrm{HR}$, TACE-RFA, and TACE in regulating MFS, a retrospective analysis was carried out on HCC patients without MVI. Each patient was treated by one of these three procedures at the Beijing Ditan Hospital of Capital Medical University. To minimize the potential bias in the results due to baseline confounding factors, we also analyzed propensity-score matched pairs of patients in each group.

\section{Materials and Methods}

\section{Patients and treatments}

The comparative study conducted here was a retrospective analysis. The diagnosis of HCC was based on the recommendations of the AASLD, and included magnetic resonance imaging (MRI), serum alpha-fetoprotein (AFP) levels, ultrasound, angiography, and computed tomography (CT) (7). The study included 828 patients who had undergone initial treatment with HR, TACE-RFA, TACE, or conservative therapy without any locoregional therapies or surgical treatment (control) at the Beijing Ditan Hospital (Beijing, China), Capital Medical University between October 2008 and November 2015. 158 patients were enrolled in the validation cohort from Putuo Hospital (Shanghai, China), Shanghai University of Traditional Chinese Medicine, and Second Hospital of Nanjing (Nanjing, China) between December 2014 and October 2015. The following criteria were used: (1) patients with HCC of BCLC A or B stage, and Child-Pugh class A or B; (2) patients without extrahepatic metastasis; (3) patients with etiologies of hepatitis $\mathrm{C}$ virus (HCV), hepatitis B virus (HBV), and/or related to alcohol; (4) patients with autoimmune liver disease, hepatitis A, D or E, syphilis, acquired immune deficiency syndrome were excluded; (5) patients with incomplete data or who lacked follow-up were excluded. MVI showed portal and/or hepatic vein filling defect in contrast-enhanced imaging (CT-scan or MRI), and embolic enhancement was identical or resembled to patients with primary liver cancer (23). The study was approved by each participating centre's ethics committee. All participating patients signed informed consent.

The appropriate treatment was selected by our multidisciplinary team. The following criteria were used for HR: shortage of ascites or hypersplenism, sufficient residual liver confirmed using volumetric computed tomography (CT), and Child-Pugh class A or selected B liver function. Indications for TACE-RFA were Child-Pugh A or B liver function, absent massive ascites or severe hypersplenism, and was performed in patients who unwilling to receive HR. Indications for TACE alone were Child-Pugh A or B liver function, absent massive ascites, or with gross classification of diffuse type.

\section{Hepatic resection procedure}

Intraoperative ultrasound was routinely performed to confirm the tumor location and to evaluate the vascular anatomy of the liver. The inflow of blood to the liver was occluded using Pringle's maneuver. The clamp crushing method was used to perform the liver resection. The resection margin exceeded $1 \mathrm{~cm}$. In addition, the detection of adequate drainage was confirmed.

\section{Radiofrequency ablation procedure}

Performed RFA within 2 weeks after TACE. Conscious analgesic sedation (intravenous administration with $0.1 \mathrm{~g}$ pethidine hydrochloride, 0.5 
$\mathrm{mg}$ atropine together with $10 \mathrm{mg}$ diazepam), and local anesthesia (5 ml lidocaine at a concentration of $1 \%$ ) were used to carry out RFA. Simultaneous RFA procedures were conducted percutaneously under nonenhanced CT (NECT) by two of four ablation experts who had 6 to 15 years of experience. The number of overlapping ablation points depended on the diameter and number of tumors, and the experts aimed to obtain an ablative margin of at least $0.5 \mathrm{~cm}$ in the normal tissues around the tumor, excluding the subcapsular portion and perivascular portions. At the end of the procedure, the experts ablated the needle tract to prevent bleeding and tumor seeding.

\section{Transcatheter arterial chemoembolization procedure}

Under local anesthesia, a catheter of $4 \mathrm{~F}-5 \mathrm{~F}$ was introduced into the abdominal aorta through the superficial femoral artery utilizing the Seldinger technique. Hepatic arterial angiography was performed under fluoroscopic guidance and the guiding catheter was directed towards the coeliac artery and superior mesenteric artery. We then identified the tumor stain, the feeding artery, as well as the vascular anatomy around the tumor. The catheter was then used to direct a microcatheter towards the feeding arteries, following which a combination of lipiodol (5-10 ml), 5-fluorouracil (50 $\mathrm{mg})$, and pirarubicin ( $30 \mathrm{mg}$ ) was introduced into the tumor. Subsequently, embolization was performed using gelatin sponge particles. An additional angiogram was performed at the end of the procedure to ensure full embolization of the supplying artery.

\section{Follow-up}

Patient MFS and OS were the endpoints of the study, which were measured in months from the time of the initial diagnosis of HCC to MVI-positive findings or death. In order to assess the technical effects, enhanced MRI or contrast-enhanced computed tomography (CECT) were performed after four weeks, and follow-ups were conducted once every three months for three years. CECT or enhanced MRI, chest CT, liver function tests, and AFP tests were performed at each visit.

During the follow-up period, patients who developed recurrences, including extrahepatic recurrence, local lesion recurrence, and intrahepatic distant recurrence, would undergo specific treatments such as RFA, tumor resection, TACE, or sorafenib administration as well as conservative treatment according to the features of the recurrent tumor, liver function status, and individual patient requirements. MVI incidence referred to the interval from the time of detection of the MVI following primary treatment to the time of death or the last follow-up date.

\section{Statistical analysis}

Continuous variables were represented by the mean $\pm \mathrm{SD}$, noncontinuous variables by the median value and range, and categorical variables by number and percentage. As the patients were not randomized to undergo HR, TACE-RFA, or TACE, the three treatment groups may have had confounding differences at baseline. Hence, we used logistic regression to generate propensity scores (PS) for all patients in order to reduce bias in our analyses. The three treatment groups were matched with the control group according to the generated propensity scores using a caliper width of 0.15 (24). On the completion of matching, the baseline covariates were compared using the paired t-test or Mann-Whitney $U$ test for continuous variables and the chi-square test for categorical variables. The Kaplan-Meier method was used to construct the MVI incidence curve which was compared using log-rank test. A two-tailed $p$ value < 0.05 was considered to be statistically significant. The above statistical analyses were conducted using SPSS for Windows 22.

\section{Results}

\section{Data of characteristics before and after propensity score matching}

A total of 1828 patients diagnosed with HCC who underwent initial treatment at the Beijing Ditan Hospital (Beijing, China), Capital Medical University between October 2008 and November 2015 were initially investigated. Of these, 701 patients had evidence of MVI, Child-Pugh class C, and/or extrahepatic metastasis. We excluded 299 patients who did not meet the abovementioned five criteria. Finally, 828 patients were included in the study, of which 116 patients underwent HR, 395 patients underwent TACE-RFA, 239 underwent TACE, and 78 patients received conservative treatment only (did not receive any locoregional therapies or surgical treatment) (Figure 1).

The HR, and TACE-RFA groups showed obvious differences compared to the control group with respect to baseline characteristics prior to PS matching. The HR group had lower gamma-glutamyl transferase (GGT) and total bilirubin (TBIL) levels than the control group $(p=0.039$ and $p=0.021)$ and younger age than the control group $(p<0.001)$. A significant difference was seen in the Model for End-Stage Liver Disease (MELD) score between the HR and control groups $(\mathrm{p}=0.005)$. TBIL level, Child-Pugh class, and prothrombin time (PT) were also significantly different between the TACE-RFA 
and control groups. In the TACE versus control group, variables were balanced at baseline (Table 1).

In the HR versus control group matched 1:1, the propensity score model included the variables of age, GGT and TBIL levels, and MELD score. In the
TACE-RFA versus control group matched 1:1, variables in the propensity score model included TBIL level, Child-Pugh class, and PT (Table 2). After PS matching, the important related characteristics became well balanced.

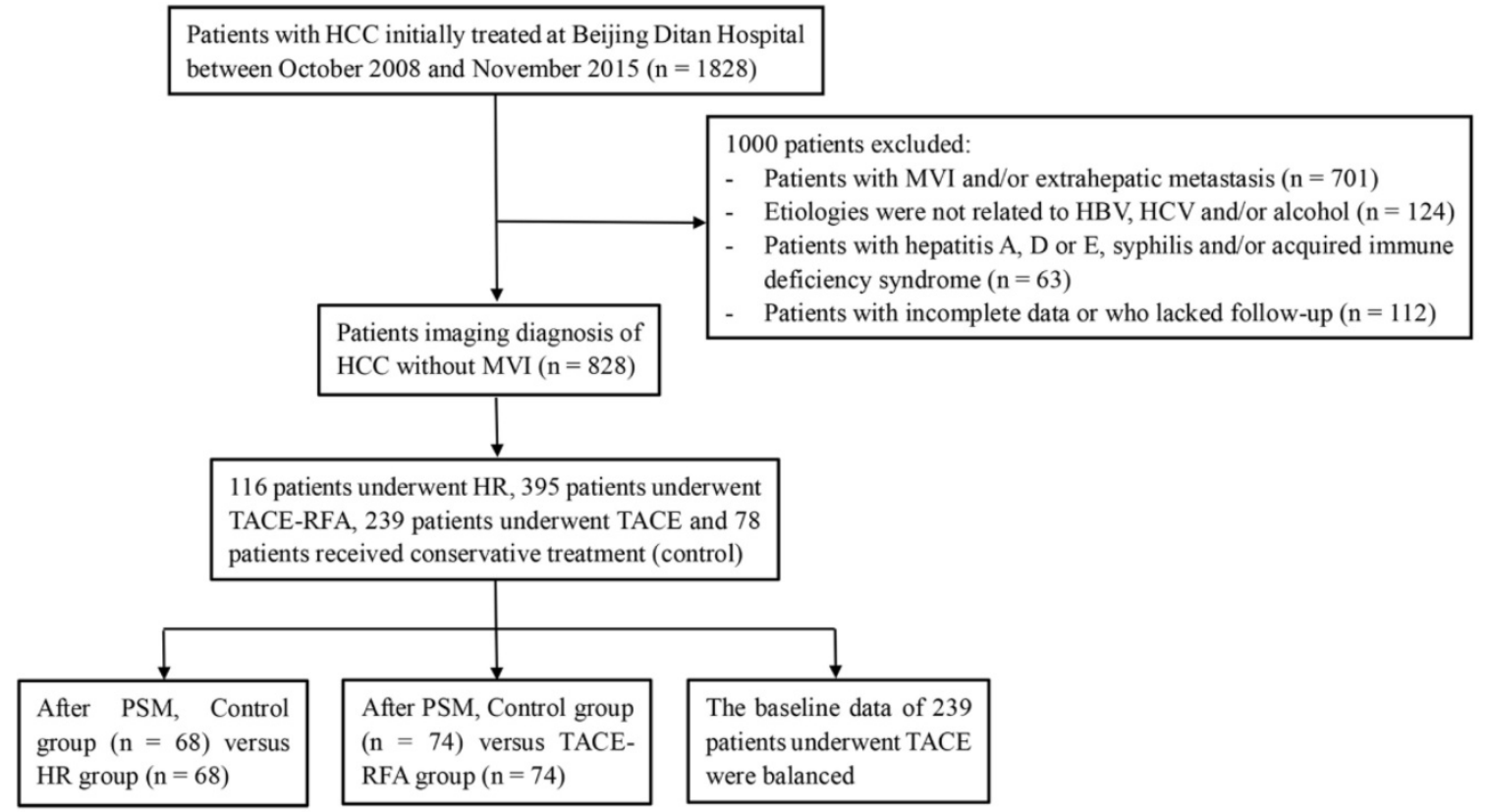

Figure 1. Flowchart of the treatments included in the study.

Table 1. Baseline characteristics of patients before matching

\begin{tabular}{|c|c|c|c|c|c|c|c|}
\hline & Control & HR & & TACE-RFA & & TACE & \\
\hline Variables & $\mathrm{n}=78$ & $\mathrm{n}=116$ & $p$ Value & $\mathrm{n}=395$ & $p$ Value & $n=239$ & $p$ Value \\
\hline Median Age (range) & $58(36-78)$ & $52(29-74)$ & $<0.001$ a) & $56(28-81)$ & 0.341 a) & $57(33-84)$ & 0.677 a) \\
\hline $\operatorname{Sex}(M / F)$ & $64 / 14$ & $96 / 20$ & $0.899 \mathrm{~b})$ & $309 / 86$ & $0.450 \mathrm{~b})$ & $195 / 61$ & $0.276^{b)}$ \\
\hline HBV related (yes/no) & $68 / 10$ & $104 / 12$ & 0.594 b) & $331 / 64$ & $0.452^{b)}$ & $214 / 42$ & $0.444^{b)}$ \\
\hline GGT (IU/L) & $52.1(27.3-117.4)$ & $36.8(22.3-80.1)$ & 0.039 c) & $46.8(26.6-88.9)$ & $0.374^{\text {c) }}$ & $46.8(26.4-87.3)$ & 0.350 c) \\
\hline TBIL $(\mu \mathrm{mol} / \mathrm{L})$ & $19.3(13.9-34.6)$ & $15.7(11.2-24.4)$ & 0.021 c) & $16.4(11.5-24.3)$ & 0.011 c) & $17.8(12.6-27.2)$ & 0.183 c) \\
\hline Child-Pugh class (A/B) & $47 / 31$ & $83 / 33$ & $0.101 \mathrm{~b})$ & $294 / 101$ & $0.011 \mathrm{~b})$ & $158 / 81$ & $0.348^{b)}$ \\
\hline PT (s) (mean \pm SD) & $13.8 \pm 2.6$ & $13.4 \pm 2.3$ & 0.238 a) & $13.1 \pm 1.9$ & 0.022 a) & $13.3 \pm 2.2$ & 0.086 a) \\
\hline MELD Score (mean $\pm S D)$ & $6.3 \pm 5.3$ & $4.3 \pm 3.5$ & 0.005 a) & $5.2 \pm 4.1$ & 0.093 a) & $5.8 \pm 4.7$ & 0.431 a) \\
\hline $\operatorname{AFP}(n g / m L)(<400 / \geq 400)$ & $60 / 18$ & $99 / 17$ & $0.135 \mathrm{~b})$ & $337 / 58$ & $0.065 \mathrm{~b})$ & $195 / 44$ & 0.367 b) \\
\hline Tumor number $(<3 / \geq 3)$ & $59 / 19$ & $90 / 26$ & 0.753 b) & $306 / 89$ & 0.725 b) & $170 / 69$ & $0.440 \mathrm{~b})$ \\
\hline Largest tumor diameter $(<5 \mathrm{~cm} / \geq 5 \mathrm{~cm})$ & $65 / 13$ & $96 / 20$ & 0.917 b) & $315 / 80$ & 0.466 b) & $179 / 60$ & 0.124 b) \\
\hline BCLC (A/B) & $51 / 27$ & $79 / 37$ & $0.693 \mathrm{~b})$ & $279 / 116$ & $0.356^{b)}$ & $144 / 95$ & 0.418 b) \\
\hline
\end{tabular}

Data are presented as mean \pm standard deviation (SD), or median (interquartile range). a) $t$ test. b) Chi-square test or Fisher's exact test. c) Mann-Whitney $U$ test. HBV, hepatitis B virus; GGT, gamma-glutamyl transferase; TBIL, total bilirubin; NLR neutrophil-lymphocyte ratio; PT, prothrombin time; MELD, Model for End-Stage Liver Disease Score; AFP, a-fetoprotein; BCLC, Barcelona Clinic for Liver Cancer.

Table 2. Characteristics of patients after matching

\begin{tabular}{|c|c|c|c|c|c|c|}
\hline & Control & HR & & Control & TACE-RFA & \\
\hline Variables & $n=68$ & $n=68$ & $P$ Value & $\mathrm{n}=74$ & $\mathrm{n}=74$ & $p$ Value \\
\hline Median Age (range) & $57(36-78)$ & $55(29-71)$ & 0.168 a) & $58(36-78)$ & $56(28-81)$ & 0.367 a) \\
\hline $\operatorname{Sex}(M / F)$ & $56 / 12$ & $55 / 13$ & $0.825 \mathrm{~b})$ & $61 / 13$ & $59 / 15$ & 0.675 b) \\
\hline HBV related (yes/no) & $60 / 8$ & $61 / 7$ & $0.784^{b)}$ & $64 / 10$ & $63 / 11$ & $0.814^{\mathrm{b})}$ \\
\hline GGT (IU/L) & $48.0(24.2-84.0)$ & $34.1(22.7-88.3)$ & 0.354 c) & $52.1(28.7-132.0)$ & $46.0(22.3-90.1)$ & $0.296^{c)}$ \\
\hline TBIL ( $\mu \mathrm{mol} / \mathrm{L})$ & $18.9(13.1-28.0)$ & $15.7(10.9-22.6)$ & $0.136^{c)}$ & $19.7(14.0-35.3)$ & $18.5(12.2-28.9)$ & $0.255^{c)}$ \\
\hline Child-Pugh class (A/B) & $45 / 23$ & $46 / 22$ & $0.855^{b)}$ & $44 / 30$ & $54 / 20$ & 0.082 b) \\
\hline PT (s) $($ mean $\pm S D)$ & $13.0(11.7-14.3)$ & $12.8(11.7-13.9)$ & 0.462 c) & $13.4(12.4-15.1)$ & $13.0(12.1-14.7)$ & $\left.0.611^{c}\right)$ \\
\hline MELD Score (mean $\pm S D)$ & $5.5 \pm 4.5$ & $5.0 \pm 3.2$ & 0.436 a) & $6.4 \pm 5.4$ & $5.7 \pm 4.6$ & 0.412 a) \\
\hline $\operatorname{AFP}(\mathrm{ng} / \mathrm{mL})(<400 / \geq 400)$ & $54 / 12$ & $59 / 9$ & 0.431 b) & $57 / 17$ & $63 / 12$ & 0.282 b) \\
\hline Tumor number $(<3 / \geq 3)$ & $49 / 19$ & $52 / 16$ & 0.556 b) & $55 / 19$ & $58 / 16$ & 0.562 b) \\
\hline Largest tumor diameter $(<5 \mathrm{~cm} / \geq 5 \mathrm{~cm})$ & $56 / 12$ & $56 / 12$ & $1.000^{b)}$ & $61 / 13$ & $58 / 16$ & 0.534 b) \\
\hline BCLC (A/B) & $44 / 24$ & $46 / 22$ & 0.717 b) & $47 / 27$ & $51 / 23$ & 0.487 b) \\
\hline
\end{tabular}



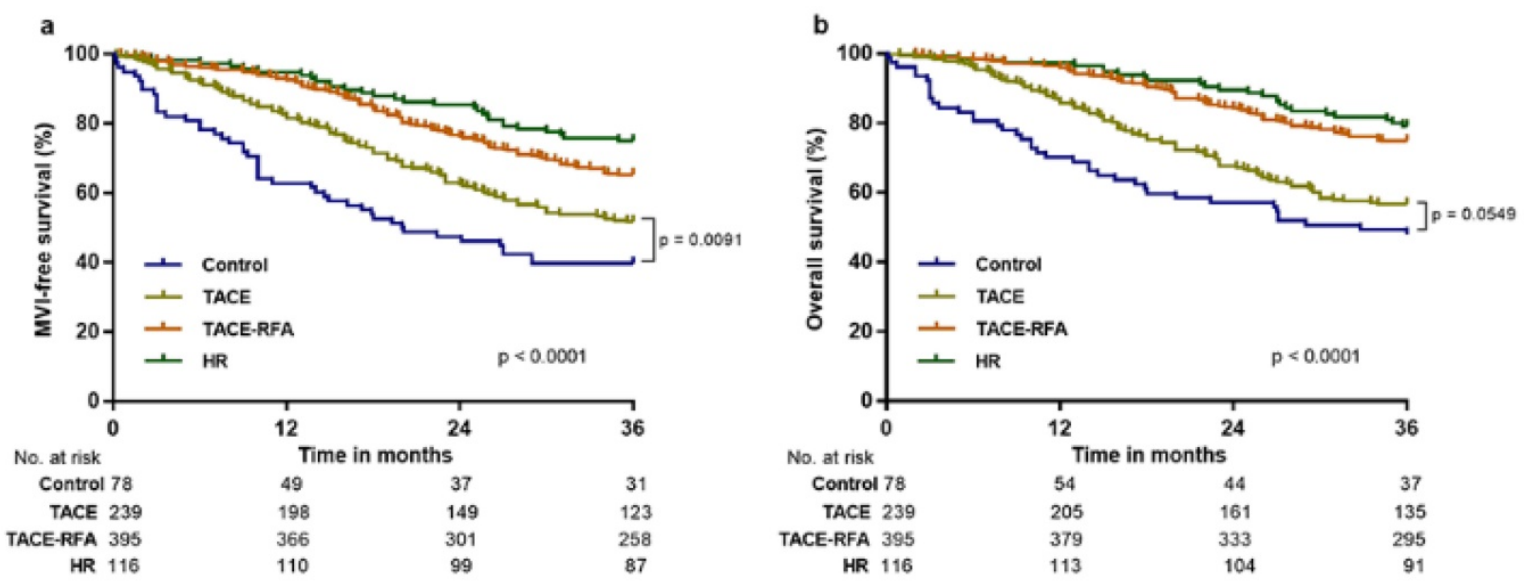

Figure 2. Macrovascular invasion-free survival (MFS) and overall survival (OS) in patients with hepatocellular carcinoma following various methods of treatment before propensity score (PS) matching analysis. The MFS and OS associated with hepatic resection (HR), transcatheter arterial chemoembolization (TACE) with radiofrequency ablation (TACE-RFA), TACE, and control treatment at 36 months were $75.0 \%$ and $78.4 \%, 65.3 \%$ and $74.7 \%, 51.5 \%$ and $56.5 \%, 39.7 \%$ and $47.4 \%$, respectively.

\section{Analysis of the MFS and OS}

During the three-year follow-up period, 87 out of $116(75 \%)$ patients in the HR group, 258 out of 395 (65.3\%) patients in the TACE-RFA group, 123 out of $239(51.5 \%)$ patients in the TACE group, and 31 out of $78(39.7 \%)$ patients in the control group showed MFS (Figure 2a), and 91 out of $116(78.4 \%)$ patients in the HR group, 295 out of $395(74.7 \%)$ patients in the TACE-RFA group, 135 out of $239(56.5 \%)$ patients in the TACE group, and 37 out of $78(47.4 \%)$ patients in the control group survived (Figure 2b). Before PS matching, Kaplan-Meier analysis showed that the HR and control groups exhibited significant difference in MFS and OS ( $p<0.0001$ for all; Figure 2). Furthermore, MFS and OS were significantly different between the TACE-RFA and control groups ( $\mathrm{p}<$ 0.0001 for all; Figure 2). No significant difference was observed between the TACE and control groups for OS at the three-year follow-up ( $p=0.0549$; Figure $2 b$ ), and MFS was obviously higher in the TACE group $(p$ $=0.0091$, Figure 2a).

PS matching was employed to generate 68 pairs of patients in the control versus HR group (1-to-1 matched), for which MFS and OS were significantly higher in the HR group than in control group after three years $(p<0.0001$ and $p=0.0010$, respectively; Figure $3 a$ and $3 b$ ). MFS and OS were also significantly higher in the TACE-RFA group than in the control group after three years $(\mathrm{p}=0.0001$ and $\mathrm{p}<0.0001$, respectively; Figure $3 c$ and $3 d$ ) in the 74 pairs of patients in the control versus TACE-RFA group (1-to-1 matched).

Furthermore, we analyzed the three-year MFS and OS to compare the effects of the treatments of HR, TACE-RFA and TACE. The baseline characteristics for the three treatment groups were similar. The results confirmed no significant difference between the HR group and TACE-RFA group with respect to
MFS and OS $(p=0.6163$ and $p=0.5003$, respectively; Figure 4). However, the HR and TACE-RFA groups had significantly higher MFS and OS than the TACE group (Figure 4).

\section{Subgroup analysis}

We further analyzed the three-year MFS and OS in HCC patients with BCLC A or B stage who underwent HR or TACE-RFA. Our results demonstrated that the three-year MFS was similar in the BCLC A (Figure 5a) or B (Figure 5b) stages after HR or TACE-RFA $(p=0.6543$ and $p=0.1289$, respectively), and that the three-year OS was also similar in the BCLC A (Figure $5 \mathrm{c}$ ) or B (Figure $5 \mathrm{~d}$ ) stages after HR or TACE-RFA $(p=0.0816$ and $p=$ 0.1975 , respectively).

\section{External validation cohort}

158 patients were included in the external validation cohort, of which 33 patients underwent HR, 35 patients underwent TACE-RFA, 48 underwent TACE, and 42 patients received conservative treatment. After PS matching, MFS and OS were significantly higher in the HR, TACE-RFA or TACE groups than in the control group after three years $(p<$ 0.0001 for all), and MFS and OS were significantly higher in the HR or TACE-RFA groups than in the TACE group (Figure 6a-6d). There was no significant difference between the HR and TACE-RFA groups with respect to MFS and OS $(p=0.8326$ and $p=$ 0.9558 , respectively; Figure 6e and $6 \mathrm{f}$ ). The above results confirmed that the effects of different treatments in the external validation cohort were similar to those in the derivation cohort.

\section{Discussion}

MVI is a common complication of HCC at advanced stages and shows a close association with 
intrahepatic metastasis and recurrence after transplantation, resulting in a poor prognosis (25-27). The focus of clinical investigations is aimed at the safety and survival outcomes of RFA, TACE and HR for patients with HCC. However, the role of treatment and management of HCC on the occurrence of MVI still remains unknown.

In the present study, we retrospectively enrolled 828 patients initially treated at our hospital with HR, TACE-RFA, TACE, and conservative therapy. The 3 -year MFS of HCC patients treated with HR and TACE-RFA was approximately $75 \%$ and $65.3 \%$,
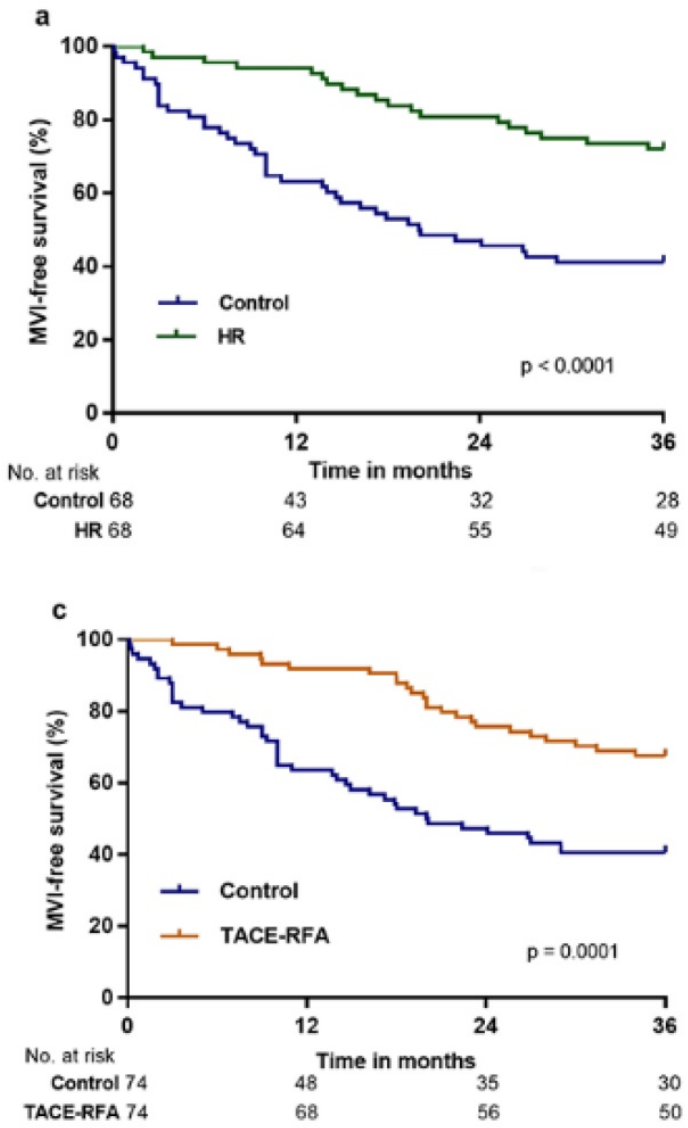

respectively, which was more satisfactory than the effect of TACE (51.5\%) alone or control (39.7\%). Hence, HR or TACE-RFA may reduce the occurrence of MVI and indirectly lengthen the survival time of HCC patients. After using PS matching to generate patient pairs that exhibited no significant differences at in the baseline, the results confirmed that HR or TACE-RFA may similarly increase the MFS and OS, which was confirmed using the external validation cohort.
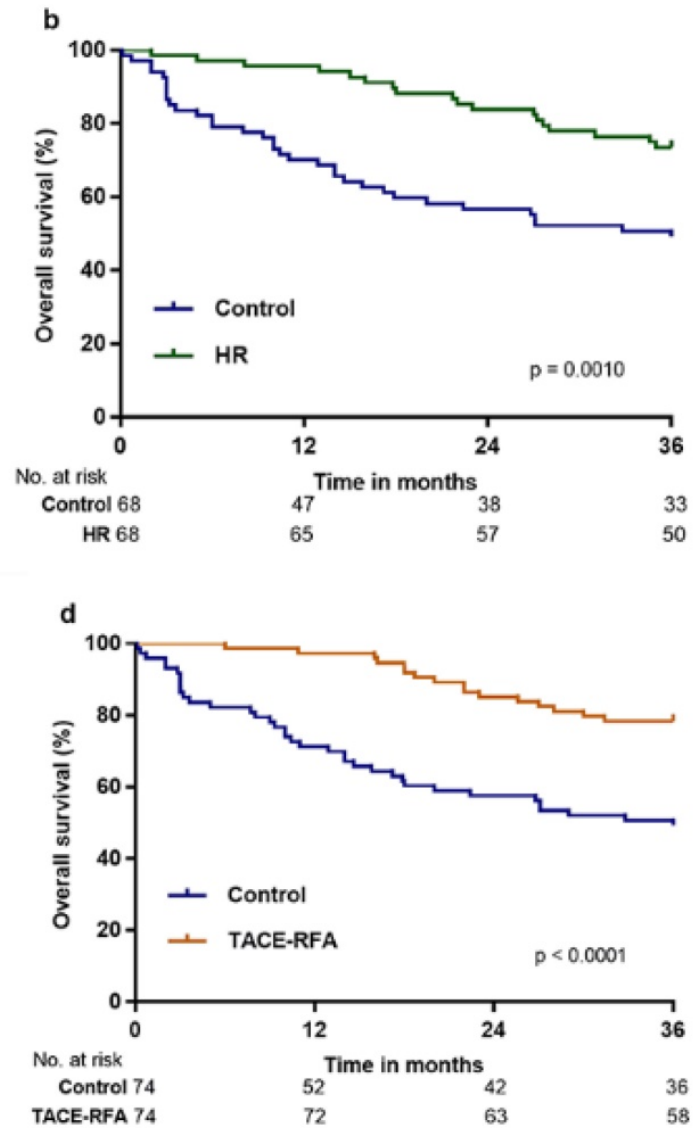

Figure 3. MFS and OS of patients in the HR, TACE-RFA, and TACE groups compared with those in the control group after PS matching analysis: a, MFS associated with HR vs. control; b, OS associated with HR vs. control; c, MFS associated with TACE-RFA vs. control; d, OS associated with TACE-RFA vs. control.
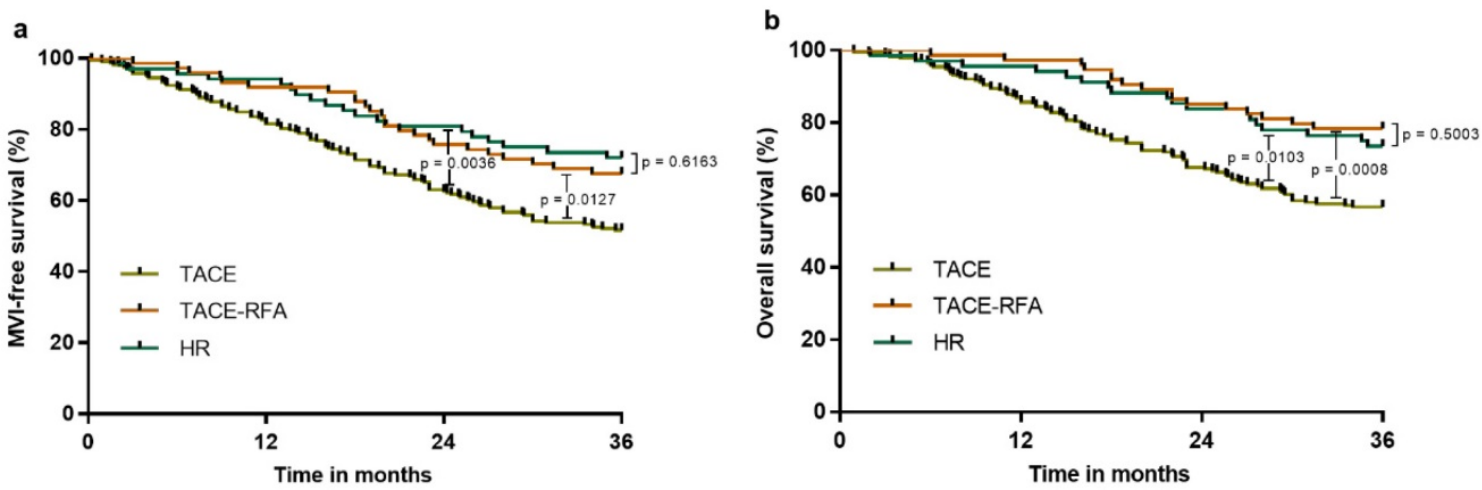

Figure 4. MFS and OS of patients in the HR vs. TACE-RFA vs. TACE groups after PS matching: a, MFS associated with HR vs. TACE-RFA vs. TACE; b, OS associated with HR vs. TACE-RFA vs. TACE. 
Nowadays, there are various guidelines for treating HCC. The recommended treatments for early HCC based on the Milan criteria show a relative consistency. Typically, HR can contribute to a 5-year survival rate $>50 \%$ for patients suffering early-stage HCC who satisfy the Milan criteria; thus, it is defined by the BCLC staging system as a preferred treatment $(28,29)$. However, a large number of studies that compared the therapeutic efficacy of HR and RFA reported a similar efficacy regarding the survival outcomes for a single small HCC of $\leq 3 \mathrm{~cm}$ (30-33). Moreover, several studies concluded that the survival rate was higher with $\mathrm{HR}$ than with TACE for patients suffering BCLC stage A and B HCC (34-37). However, treatments are controversial for patients with multinodular tumors or those at BCLC C stage, even if the tumors are potentially resectable.

The study aimed to compare the effects of various treatment modalities on three-year MFS, which could significantly influence the median survival time of patients with HCC. We used patients undergoing conservative treatment as the control group, with a BCLC stage of 0-D. After PS matching with the conservative treatment group, the important factors of baseline such as liver functions and BCLC
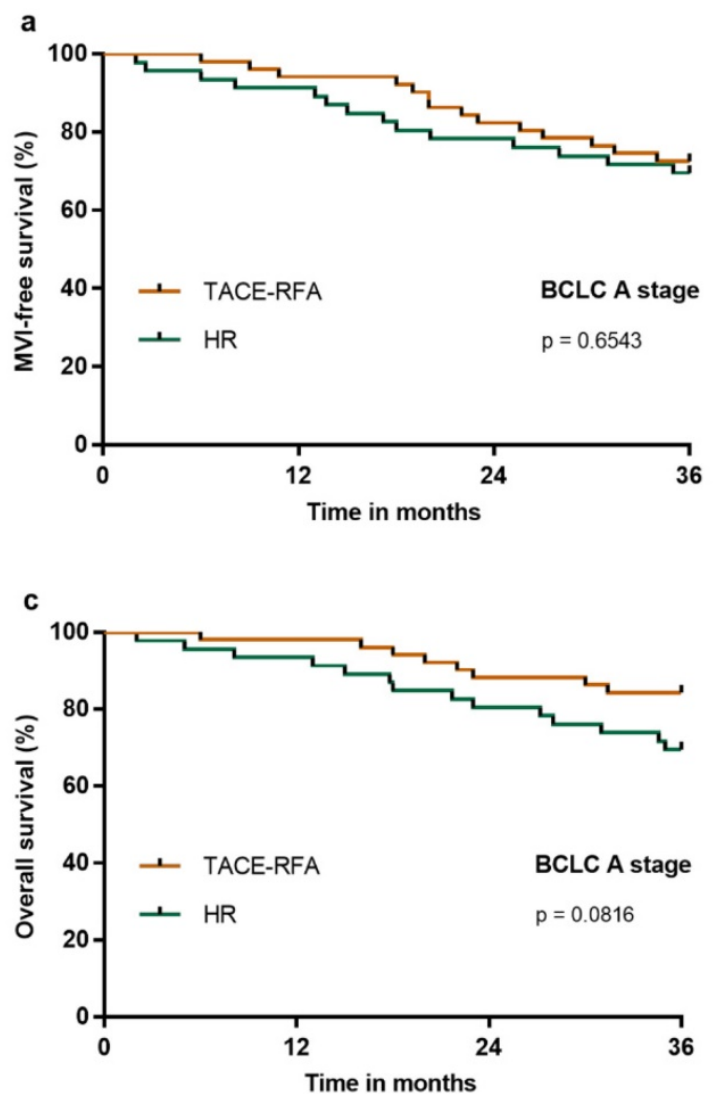

staging were well balanced; the results showed that HR and TACE-RFA can similarly increase the MFS and $O S$ at the 3-year follow-up. The results were confirmed after PS matching with HR group versus TACE-RFA group. This may be the reason that HR or TACE-RFA contributed to a higher survival rate than TACE for HCC patients.

Our study had a few limitations. First, the etiologies of patients we enrolled were $\mathrm{HBV}, \mathrm{HCV}$, and/or alcohol-related; we did not account for the possible differences in the prevalence for each treatment part or the differences in virus activity, history of antiretroviral therapy, or alcohol level intake. Second, we did not take into account the possible differences between TACE patients based on the number of treatment cycles they underwent. A previous study suggested that the OS was significantly higher for patients receiving $\geq 3$ cycles of TACE than for those receiving $<3$ cycles (38). Finally, the retrospective nature of our study raised the risk of confounding, despite our use of PS matching. The study results should be modified based on the results from randomized studies that better consider patient populations at different sites.

b

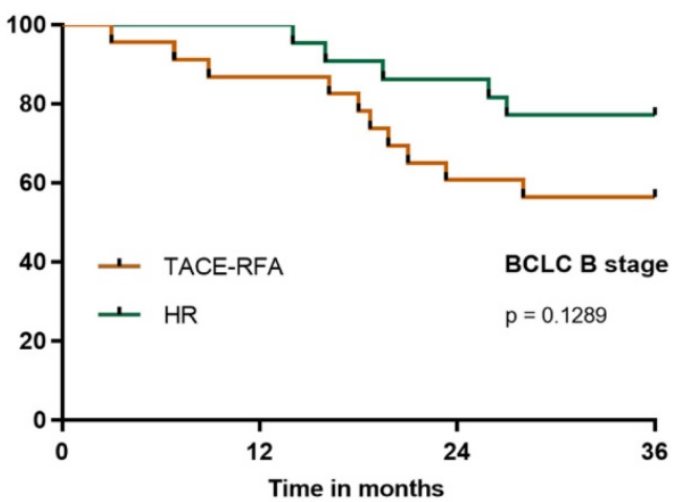

d

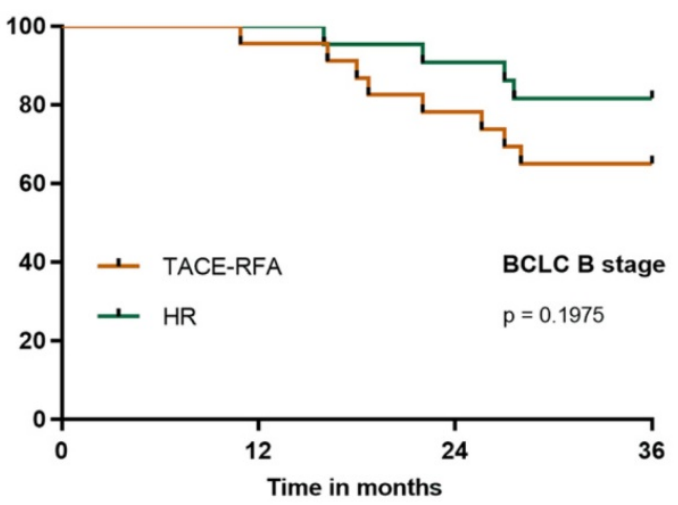

Figure 5. MFS and OS of patients in the HR group compared with the TACE-RFA group after PS matching analysis in BCLC A or B stages: a, MFS in BCLC A stage; b, MFS in BCLC B stage; c, OS in BCLC A stage; b, OS in BCLC B stage. 

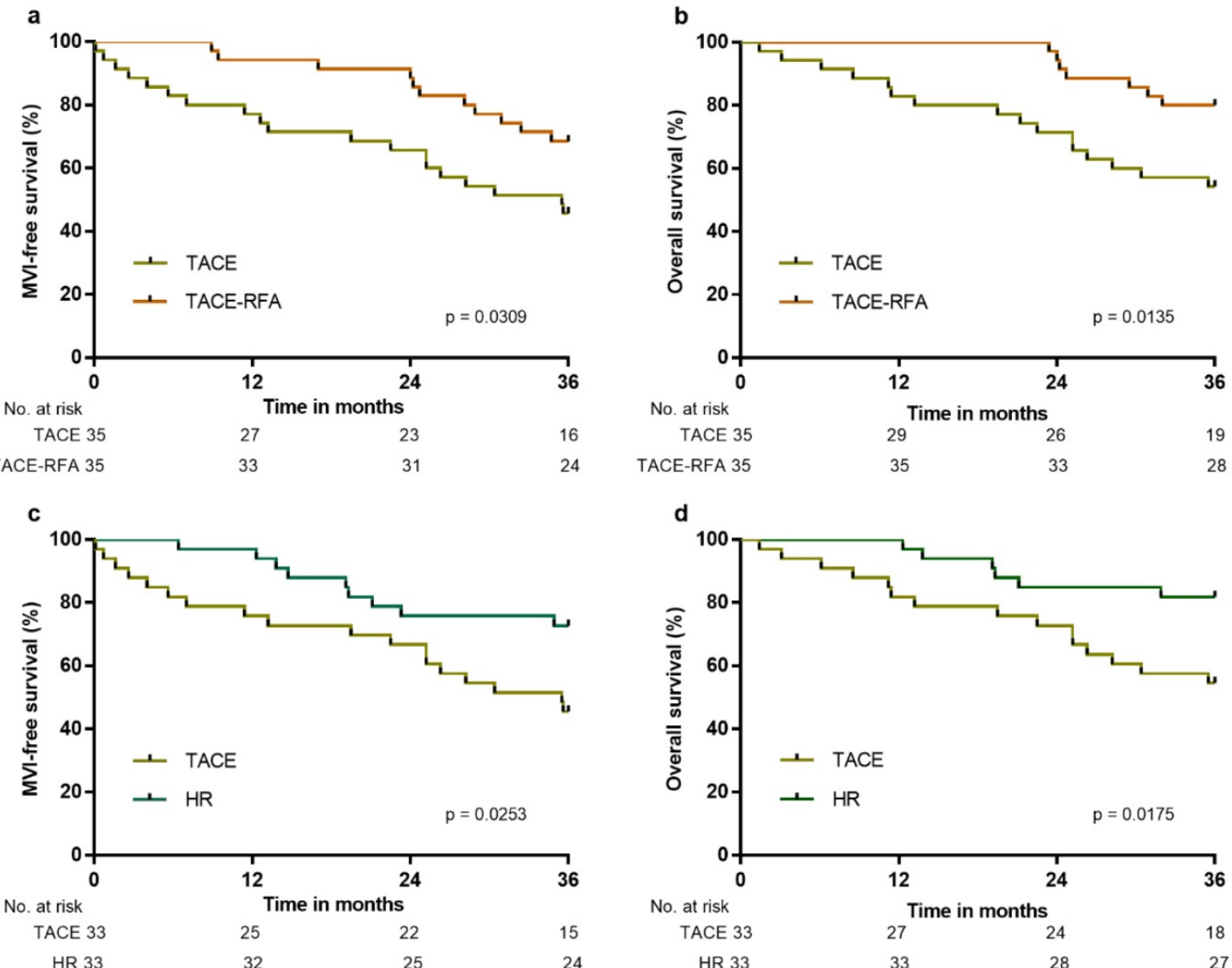

d

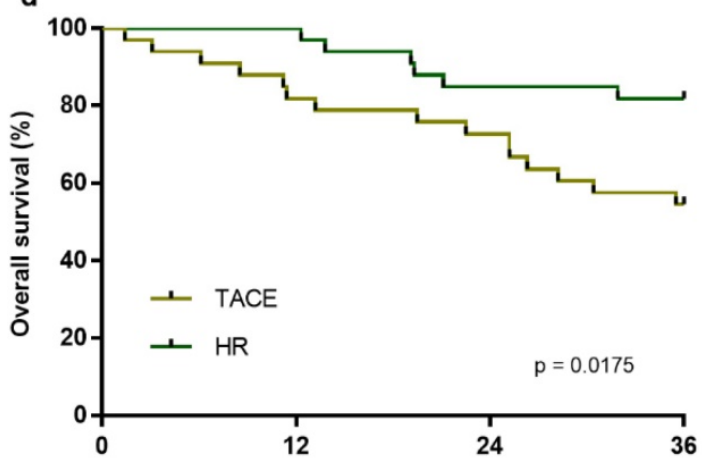

No. at risk

TACE 33

Time in months

HR 33

24
28

f
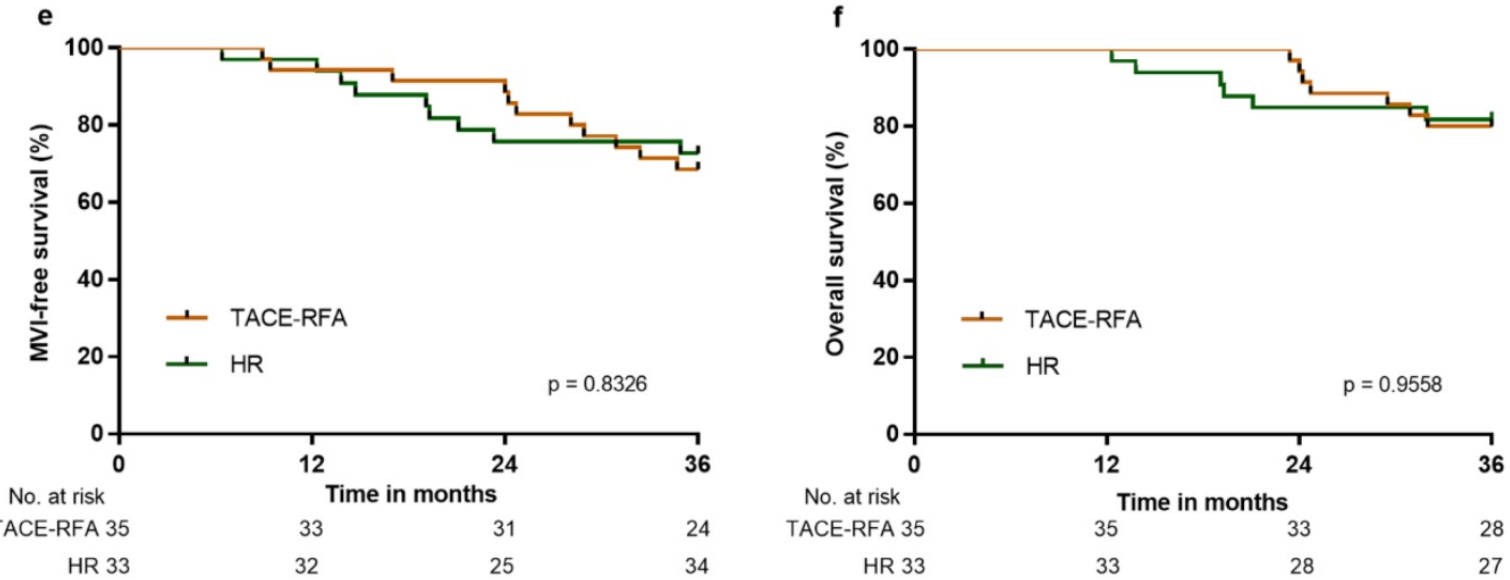

No. at risk

TACE-RFA 35

Time in months

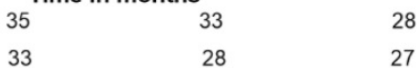

Figure 6. MFS and OS of patients in the external validation cohort: a, MFS associated with TACE-RFA vs. TACE; b, OS associated with TACE-RFA vs. TACE; c, MFS associated with HR vs. TACE; d, OS associated with HR vs. TACE; e, MFS associated with HR vs. TACE-RFA; $f$, OS associated with HR vs. TACE-RFA.

\section{Conclusions}

Our study confirms that the higher MFS and OS obtained using HR or TACE-RFA rather than TACE for HCC patients without MVI may help guide treatment decisions.

\section{Acknowledgements}

This study was completed with the support of the National Natural Science Foundation of China under Grant No. 81473641 and 81774234, the Beijing Municipal Administration of Hospitals Clinical Medicine Development of Special Funding (No. ZYLX201707) and the Capital's Funds for Health Improvement and Research (No. 2018-1-2172).

\section{Competing Interests}

The authors have declared that no competing interest exists. 


\section{References}

1. Miller KD, Siegel RL, Lin CC, Mariotto AB, Kramer JL, Rowland JH, Stein KD, et al. Cancer treatment and survivorship statistics, 2016. CA Cancer J Clin. 2016;66:271-289.

2. Cheung TK, Lai CL, Wong BC, Fung J, Yuen MF. Clinical features, biochemical parameters, and virological profiles of patients with hepatocellular carcinoma in Hong Kong. Aliment Pharmacol Ther. 2006;24:573-583.

3. Minagawa M, Makuuchi M. Treatment of hepatocellular carcinoma accompanied by portal vein tumor thrombus. World J Gastroenterol. 2006;12:7561-7567

4. Llovet JM, Bustamante J, Castells A, Vilana R, Ayuso Mdel C, Sala M, Bru C, et al. Natural history of untreated nonsurgical hepatocellular carcinoma: rationale for the design and evaluation of therapeutic trials. Hepatology. 1999;29:62-67.

5. Schoniger-Hekele M, Muller C, Kutilek M, Oesterreicher C, Ferenci P, Gangl A. Hepatocellular carcinoma in Central Europe: prognostic features and survival. Gut. 2001;48:103-109.

6. EASL-EORTC clinical practice guidelines: management of hepatocellular carcinoma. J Hepatol. 2012;56:908-943.

7. Bruix J, Sherman M. Management of hepatocellular carcinoma: an update. Hepatology. 2011;53:1020-1022.

8. Mazzaferro V, Regalia E, Doci $R$, Andreola S, Pulvirenti A, Bozzetti $F$, Montalto F, et al. Liver transplantation for the treatment of small hepatocellular carcinomas in patients with cirrhosis. $N$ Engl J Med. 1996;334:693-699.

9. Poon RT, Fan ST, Lo CM, Liu CL, Wong J. Difference in tumor invasiveness in cirrhotic patients with hepatocellular carcinoma fulfilling the Milan criteria treated by resection and transplantation: impact on long-term survival. Ann Surg. 2007;245:51-58.

10. Concejero A, Chen CL, Wang CC, Wang SH, Lin CC, Liu YW, Yang CH, et al. Living donor liver transplantation for hepatocellular carcinoma: a single-center experience in Taiwan. Transplantation. 2008;85:398-406.

11. Turcotte S, Dematteo RP. Resection versus transplantation for early hepatocellular carcinoma: more art than science. Ann Surg. 2012;256:892-893.

12. Chan SC. Liver transplantation for hepatocellular carcinoma. Liver Cancer. 2013;2:338-344.

13. Kagawa T, Koizumi J, Kojima S, Nagata N, Numata M, Watanabe N, Watanabe $\mathrm{T}$, et al. Transcatheter arterial chemoembolization plus radiofrequency ablation therapy for early stage hepatocellular carcinoma: comparison with surgical resection. Cancer. 2010;116:3638-3644.

14. Yamakado K, Nakatsuka A, Takaki H, Yokoi H, Usui M, Sakurai H, Isaji S, et al. Early-stage hepatocellular carcinoma: radiofrequency ablation combined with chemoembolization versus hepatectomy. Radiology. 2008;247:260-266.

15. Hsu KF, Chu CH, Chan DC, Yu JC, Shih ML, Hsieh HF, Hsieh TY, et al. Superselective transarterial chemoembolization vs hepatic resection for resectable early-stage hepatocellular carcinoma in patients with Child-Pugh class a liver function. Eur J Radiol. 2012;81:466-471.

16. Kim HC, Suk KT, Kim DJ, Yoon JH, Kim YS, Baik GH, Kim JB, et al. Transarterial chemoembolization in Barcelona Clinic Liver Cancer Stage 0/A hepatocellular carcinoma. World J Gastroenterol. 2014;20:745-754

17. Bargellini I, Sacco R, Bozzi E, Bertini M, Ginanni B, Romano A, Cicorelli A, et al. Transarterial chemoembolization in very early and early-stage hepatocellular carcinoma patients excluded from curative treatment: a prospective cohort study. Eur J Radiol. 2012;81:1173-1178.

18. Golfieri R, Cappelli A, Cucchetti A, Piscaglia F, Carpenzano M, Peri E, Ravaioli $\mathrm{M}$, et al. Efficacy of selective transarterial chemoembolization in inducing tumor necrosis in small $(<5 \mathrm{~cm})$ hepatocellular carcinomas. Hepatology. 2011;53:1580-1589.

19. Llovet JM, Bruix J. Systematic review of randomized trials for unresectable hepatocellular carcinoma: Chemoembolization improves survival. Hepatology. 2003;37:429-442.

20. Bruix J, Sherman M. Management of hepatocellular carcinoma. Hepatology. 2005;42:1208-1236

21. Guo Z, Zhong Y, Hu B, Jiang JH, Li LQ, Xiang BD. Hepatic resection or transarterial chemoembolization for hepatocellular carcinoma within Milan criteria: A propensity score matching analysis. Medicine (Baltimore). 2017;96:e8933.

22. Peng Z, Wei M, Chen S, Lin M, Jiang C, Mei J, Li B, et al. Combined transcatheter arterial chemoembolization and radiofrequency ablation versus hepatectomy for recurrent hepatocellular carcinoma after initial surgery: a propensity score matching study. Eur Radiol. 2018; 28(8):3522-3531.

23. Shah ZK, McKernan MG, Hahn PF, Sahani DV. Enhancing and expansile portal vein thrombosis: value in the diagnosis of hepatocellular carcinoma in patients with multiple hepatic lesions. AJR Am J Roentgenol. 2007;188:1320-1323.

24. D'Agostino RB, Jr. Propensity score methods for bias reduction in the comparison of a treatment to a non-randomized control group. Stat Med. 1998;17:2265-2281.

25. Pirisi M, Avellini C, Fabris C, Scott C, Bardus P, Soardo G, Beltrami CA, et al. Portal vein thrombosis in hepatocellular carcinoma: age and sex distribution in an autopsy study. J Cancer Res Clin Oncol. 1998;124:397-400.

26. Shah SA, Tan JC, McGilvray ID, Cattral MS, Levy GA, Greig PD, Grant DR. Does microvascular invasion affect outcomes after liver transplantation for
HCC? A histopathological analysis of 155 consecutive explants. J Gastrointest Surg. 2007;11:464-471.

27. Marsh JW, Dvorchik I, Bonham CA, Iwatsuki S. Is the pathologic TNM staging system for patients with hepatoma predictive of outcome? Cancer. 2000;88:538-543.

28. Santambrogio R, Opocher E, Costa M, Barabino M, Zuin M, Bertolini E, De Filippi F, et al. Hepatic resection for "BCLC stage A" hepatocellular carcinoma. The prognostic role of alpha-fetoprotein. Ann Surg Oncol. 2012;19:426-434.

29. Yamamoto J, Kosuge T, Saiura A, Sakamoto Y, Shimada K, Sano T, Takayama $\mathrm{T}$, et al. Effectiveness of hepatic resection for early-stage hepatocellular carcinoma in cirrhotic patients: subgroup analysis according to Milan criteria. Jpn J Clin Oncol. 2007;37:287-295.

30. Feng K, Yan J, Li X, Xia F, Ma K, Wang S, Bie P, et al. A randomized controlled trial of radiofrequency ablation and surgical resection in the treatment of small hepatocellular carcinoma. J Hepatol. 2012;57:794-802.

31. Kang TW, Kim JM, Rhim H, Lee MW, Kim YS, Lim HK, Choi D, et al. Small Hepatocellular Carcinoma: Radiofrequency Ablation versus Nonanatomic Resection--Propensity Score Analyses of Long-term Outcomes. Radiology. 2015;275:908-919.

32. Wang Y, Luo Q, Li Y, Deng S, Wei S, Li X. Radiofrequency ablation versus hepatic resection for small hepatocellular carcinomas: a meta-analysis of randomized and nonrandomized controlled trials. PLoS One. 2014;9:e84484.

33. Pompili M, Saviano A, de Matthaeis N, Cucchetti A, Ardito F, Federico B, Brunello $\mathrm{F}$, et al. Long-term effectiveness of resection and radiofrequency ablation for single hepatocellular carcinoma $</=3 \mathrm{~cm}$. Results of a multicenter Italian survey. J Hepatol. 2013;59:89-97.

34. D'Avola D, Inarrairaegui M, Pardo F, Rotellar F, Marti P, Bilbao JI, Martinez-Cuesta A, et al. Prognosis of hepatocellular carcinoma in relation to treatment across BCLC stages. Ann Surg Oncol. 2011;18:1964-1971.

35. Guo Z, Zhong JH, Jiang JH, Zhang J, Xiang BD, Li LQ. Comparison of survival of patients with BCLC stage A hepatocellular carcinoma after hepatic resection or transarterial chemoembolization: a propensity score-based analysis. Ann Surg Oncol. 2014;21:3069-3076.

36. Lin CT, Hsu KF, Chen TW, Yu JC, Chan DC, Yu CY, Hsieh TY, et al. Comparing hepatic resection and transarterial chemoembolization for Barcelona Clinic Liver Cancer (BCLC) stage B hepatocellular carcinoma: change for treatment of choice? World J Surg. 2010;34:2155-2161.

37. Rahbari NN, Mehrabi A, Mollberg NM, Muller SA, Koch M, Buchler MW, Weitz J. Hepatocellular carcinoma: current management and perspectives for the future. Ann Surg. 2011;253:453-469.

38. Zhao YN, Zhang YQ, Ye JZ, Liu X, Yang HZ, Cong FY, Xiang BD, et al. Hepatic resection versus transarterial chemoembolization for patients with Barcelona Clinic Liver Cancer intermediate stage Child-Pugh A hepatocellular carcinoma. Exp Ther Med. 2016;12:3813-3819. 